\title{
Fabio Armand
}

\section{Aufhocker : Punnd l'identité Alien d'un de nos corps-fantômes se porte sur le dos}

\section{AufHOCKER: THE ALIEN IDENTITY OF ONE OF OUR PHANTOM-BODIES CARRIED ON THE BACK}

Abstract: Within the framework of the most recent advances in the BRAINCUBUS model of neurocognitive anthropology, we will analyze the transcultural narrative motif of the Aufhocker (F472. Huckauf. A goblin which jumps on one's back). From the Alps to the Himalayas, we will track down experiential encounters with numerous supernatural beings who jump on the backs of humans and are carried away with all their crushing heaviness. We recognize these supernatural beings as neurally real phantom-body connectomes, generated by the activation of the Temporo-Parietal Junction in the left hemisphere of the human brain.

Keywords: Aufhocker; Experiential Narrative Imaginaries; Neurocognitive Anthropology; Alien Identity; Intentionality; Agentivity; Temporo-Parietal Junction.

\section{FABIO ARMAND}

Institut Pierre Gardette, Pôle « Culture(s), Langue et Imaginaires », UR « Confluences : Sciences et Humanités », Université Catholique de Lyon armand.fabio@gmail.com

DOI: 10.24193/cechinox.2021.41.07

\section{De l'altérité à l'identité : pour une anthropologie des} ontologies des imaginaires humains

$\mathrm{D}$ ans le cadre des plus récentes avancées du modèle d'anthropologie neurocognitive BRAINCUBUS (Armand \& Cathiard, 2019 ; Armand et al., 2019), présentées lors du $3^{\mathrm{e}}$ Congrès International du CRI2i, à Hammamet, nous nous sommes penché sur la question de l'identité Alien des corps fantômes qui hantent les imaginaires narratifs humains. En proposant une anthropologie neurocognitive de l'altérité, nous avions abordé la figure himalayenne de la Kichkannī et de ses homologues autostoppeuses fantômes alpines, en soulignant leur nature fondamentalement neurale. Si nous avions mis principalement en évidence l'expérience vécue du point de vue des victimes de ces Autres spectrales, cette étude veut contribuer à enrichir les réflexions sur la nature de ces êtres surnaturels, afin de déceler leur identité neurocognitive. Nous tournerons ainsi notre attention vers les êtres fantastiques de type Aufhocker (Motif-Index ${ }^{1}$ : F472. Huckauf. A goblin which jumps on one's back), à savoir ces ontologies surnaturelles qui sautent sur le dos d'humains et se laissent transporter de toute leur lourdeur écrasante ${ }^{2}$. 
Nous proposerons un parcours transculturel en narration qui se développera en partant, d'une catégorie spécifique de loups-garous aquatiques (Armand 2013, 2016) qui sautent sur le dos de leurs victimes humaines pour les diriger, contre leur volonté, vers une étendue d'eau où, dans le pire des cas, elles seront noyées (cf. F420.5.2.10. Water-man sits on back of persons as heavy burden). En suivant les découvertes du folklore allemand, au carrefour entre le Volkskunde et l'Erzählforschung depuis au moins le début du $\mathrm{XIX}^{\mathrm{e}}$ siècle, nous ferons appel à quelques réflexions étiologiques naïves pour analyser ces narrations. Enfin, en passant par l'Orient arabo-persan, avec ses mirabilia traversées par Alexandre le Grand lors de ses pérégrinations, nous rencontrerons des djinns spécifiques à la nature tout au moins étrange - le duwal-pāy, en persan, ou, dans la forme arabe, duwal-bāy - qui nous accompagnerons jusqu'à l'Himalaya népalais, où notre Kichannī se présentera encore une fois dans sa fonction d'Aufhocker (E262. Ghost rides on man's back).

Afin d'établir les bases pour une identité neurale de ces êtres surnaturels, nous les considérons comme des connectomes de corps-fantômes neuralement bien réels, présents dans le syndrome de la narcolepsie et, plus généralement, dans son syndrome ancillaire, la paralysie du sommeil (Abry, 2011 ; Cathiard \& Armand, 2014). La stimulation corticale a révélé que la composante généralement qualifiée de "feeling of a presence ", que nous avons identifiée plus précisément - quand elle se produit $a$ tergo - comme AP3S (Alien Presence Sensed from Self Shadowing), pouvait être induite dans la Jonction Temporo-Pariétale (TPJ) de l'hémisphère gauche du cerveau humain (depuis Arzy et al., 2006). Nos réflexions permettront d'intégrer ce phénomène dans le modèle pariéto-frontal de la cognition motrice proposé par Desmurget \& Sirigu (2009 ; Desmurget et al., 2009 ; 2018). En effet, l'Aufhocker fournit certainement la documentation spécifiquement la plus abondante sur ce phénomène, comparativement à tout le patrimoine narratif expérientiel onirique de l'Humanité, à côté de celle plus connue du cauchemar, comme l'esprit oppressant le dormeur.

Pour mieux saisir l'identité de ces ontologies de l'imaginaire dans le domaine des dissociations corporelles, nous focaliserons notre attention sur la notion d'agentivité afin de pourvoir déceler la dichotomie fondamentale entre Self agentif et Alien contre-agentif. Nous pourrons ainsi questionner la nature de cet Autre Alien qui n'est pas reconnu comme partie intégrante $\mathrm{du}$ Self, mais qui devient « a Being, (...) not as a mere existent thing, but an intentional Being " (Cheyne \& Girard, 2007, nous soulignons). Nous serons alors capables de lui reconnaître une identité précise, fondée sur l'intentionnalité et l'agentivité.

\section{Aufhockers en forme lupine}

T a première étape de notre parcours transculturel sur les traces des êtres fantastiques de type Aufhocker nous emmène à la rencontre, dans un milieu tout à fait particulier, d'une catégorie de loups-garous (D113.1.1. Werwolf. A man changes periodically into the form of a wolf. He is usually malevolent when in wolf form) qui semble apprécier tout particulièrement l'élément aquatique, véritable vecteur de leur métamorphose (Armand 2013). Ainsi, dans le département de la Creuse, 
Aufhocker : Quand l'identité Alien d'un de nos corps-fantômes se porte sur le dos

les loups-garous de la Montagne Noire devaient $[\ldots]$, au commencement et à la fin de leur course, se plonger dans les fontaines. À Guéret, la fontaine Piquerelle est un endroit choisi par le loup-garou pour guetter sa proie ; il saute sur le dos du passant et s'y cramponne si bien qu'on ne peut s'en débarrasser qu'en le blessant de façon à ce que son sang coule. (Sébillot, 1904 : 205-206)

Ces garous aquatiques se cramponnant sur le dos de leurs victimes trouvent leurs homologues dans un récit recueilli dans la "Venise du Périgord », Brantôme :

Un homme revenait tard de la foire de Brantôme lorsque, en pleine campagne, il sentit brusquement quelque chose lui tomber sur les épaules. C'était mou, lourd et cela respirait comme un être vivant. Malgré tous ses efforts pour s'en débarrasser il ne put y parvenir et eut bien du mal à redresser sa marche, car la chose le forçait à se diriger vers la rivière toute proche. Il finit par arriver chez lui, harassé comme s'il avait couru depuis son départ. Sa femme lui apprit qu'il avait dû porter un lèbérou repu, et se signa en pensant combien la mort s'était approché de son homme. (Seignolle, 1998 : 448)

En mettant de côté lélément aquatique médiateur de ces métamorphoses lycanthropiques, nous focalisons notre attention sur l'action accomplie par ces êtres fantastiques qui s'accrochent magiquement au dos de leurs victimes (cf.
G311. Old man of the sea. Burr-woman. Ogre who jumps on one's back and sticks there magically) et, les opprimant d'un poids écrasant (cf. F420.5.2.10. Water-man sits on back of persons as heavy burden), prennent le contrôle de leurs corps pour les diriger où ils désirent.

En nous déplaçant dans la Basse Normandie, nous rencontrons une autre figure particulière de varou. Dans le cadre d'une procédure judiciaire, si quelqu'un refuse de répondre aux injonctions des monitoires - des ordonnances rendues par les juges ecclésiastiques, portant injonction, sous peines d'excommunication majeure, à ceux qui ont connaissance de quelques délits de les révéler, ou à ceux qui ont fait quelque tort au prochain de le réparer -, il peut recevoir une punition exemplaire et être obligé à " porter le varou » (D661. Transformation [in werewolf] as punish$m e n t)$. C'est ce qui s'est passé, au milieu du XVIII siècle, dans le Val Ferrand, au lieudit le Douet du Moulin, suite à un double meurtre violent : ayant refusé de répondre au monitoire, un valet de ferme appelé Gliauminot vécut l'expérience désagréable de "porter le varou » pendant un mois, jusqu’à la Chandeleur :

Il couchait habituellement dans la grange, où il sétait fait un lit dans le blé. Une nuit, comme il dormait, cétait la nuit de Noël, pendant la messe de minuit, au moment où les animaux s'agenouillent, dit-on, dans les étables, - il lui semble tout à coup que quelque chose de lourd se jette sur son dos; il se lève, ouvre la porte et voilà que, malgré lui, - il l'a assuré plus tard, - il se met à courir comme un fou à travers les mares, les cavées, les 
fondrières, les ronces et les buissons, marchant devant lui sans pouvoir s'arrêter, sans pouvoir se diriger et emporté par une force irrésistible. Arrivé à un carrefour à quatre chemins, il se sent cinglé de sept coups de fouet vigoureusement appliqués. Il en est de même à chaque carrefour, mais il ne voit personne. C'est une main invisible qui le frappe. Il se croise avec plusieurs de ses connaissances; il les reconnaît, mais elles ne le reconnaissent pas ; il veut leur parler, les sons s'arrêtent dans sa gorge, il ne peut articuler un seul mot. (Fleury, 1883 : 85-89, nous soulignons)

Ce motif narratif, très répandu dans le folklore européen, se rattache au thème de la monture du diable, où un individu ayant commis une faute ou un péché est condamné à servir, pour une période déterminée, comme monture aux démons. En Normandie et, comme nous le verrons, par la suite, en Bretagne, ce motif contamine la croyance au loup-garou : le soir même de son excommunication, Gliauminot est condamné à porter sur ses épaules le varou, cet être lourd qui prend le contrôle de ses mouvements.

Ainsi, dans la région bretonne de Saint-Malo, un homme court le guérou, en transportant une petite bête noire, diabolique, sur son dos:

D'après le conte qui suit, ceux qui courent le guérou y étaient forcés par un diable qui les accompagnait partout, mais dont ils pouvaient se débarrasser en le donnant à autrui. Il était une fois un homme qui se promenait seul dans son jardin. Tout à coup il entendit un cri, et il dit tout haut : - Tu ferais bien mieux de te taire. Aussitôt un homme qui portait sur le dos une petite bête noire accourut tout essoufflé et lui dit : — Qu'est-ce que tu me veux, toi ? - Rien, répondit l'homme tout effrayé. - Hé bien! dit le coureur de guérou, puisque tu ne me veux rien, cours le guérou à ma place. Il lui jeta sur le dos sa petite bête noire, et il disparut. La bête se colla sur le dos de l'homme si fortement qu'il ne pouvait s'en débarrasser, et il était obligé de courir le guérou. (Sébillot, 1882 : 294-296)

Pour se débarrasser de cette " petite bête noire ", il doit la lancer sur le dos d'autrui ; toutefois, un curé peut intervenir et asperger cet Aufhocker démoniaque ${ }^{4}$ avec de l'eau bénite (V132.2. Holy water disperses demons).

\subsection{Aufhockers et cauchemars dans le folklore allemand}

Tous retrouvons le motif narratif de 1 l'Aufhocker dans sa forme lycanthropique dans le folklore narratif allemand ${ }^{5}$ et dans des régions flamandes de la Belgique et des Pays-Bas (de Blécourt, 2007 : 26-28). Afin d'appréhender ce recoupement de motifs narratifs, de Blécourt affirme que les conclusions de Roeck (1967), selon lequel la figure du «back-riding werewolf » serait issue d'une désintégration des légendes lycanthropiques, sont erronées :

Roeck took the back-riding werewolf as an example of the disintegration of the werewolf legend, and of the blurring of boundaries between the werewolf and 
Aufhocker : Quand l'identité Alien d'un de nos corps-fantômes se porte sur le dos

other figures. His conclusion "that back-riding was originally no part of the werewolf legend" echoes Muller's thesis that this was a new component, taken from the nightmare. This argument is incorrect, bowever, as the nightmare is said to suffocate people when they are lying in bed, not when they are walking abroad at night. ${ }^{6}$ (de Blécourt, $2007: 27$, nous soulignons ; cf. de Blécourt, 2015 : 15)

En reconnaissant, avec de Blécourt, la dynamique de ces types de narrations et la nature composite des protagonistes, nous voulons revaloriser l'intuition de Müller (1937 : 59) sur la croyance aux loups-garous : «ce trait [du loup-garou comme Huckup] a été transposé de l'inventaire de narrations sur l'Alp [comme cauchemar] et les autres fantômes oppressants : c'est précisément sous la forme du croisement entre les motifs narratifs du Huckup et du loup-garou que ce trait apparaît très souvent aujourd'hui " (notre traduction). Ayant déjà démontré la stricte parenté neuroanthropologique entre les loups-garous et les cauchemars (Armand, 2016), nous corrigerons, du point de vue irréfutable des connectomes de corps-fantômes neuralement bien réels, l'accusation maladroite d'inexactitude énoncée si lapidairement par de Blécourt. Sans recourir à l'élément lycanthropique, Claude Lecouteux (1998 : 107), spécialiste d'études médiévales allemandes, semble bien partager nos considérations quand il confirme " les suppositions de Ranke et Höfler [voir la section suivante] ainsi que leur analyse de la Mahr en liaison avec l'Alp, le Huckup et le Aufhocker, ces deux derniers types de revenants étant l'exact pendant de l'incube ». Dans les conclusions de notre analyse, nous allons reprendre l'intuition de Müller sur le rapport établi entre les loups-garous, les cauchemars et les Aufhockers ; notre perspective se fonde sur de solides bases neurales qui démontreront clairement comment ces trois ontologies surnaturelles sont générées neuralement par les mêmes activations corticales.

\section{Une étiologie naïve autour de la figure du Aufhocker}

Comme nous l'allons vu, les premiers folkloristes allemands qui se sont penchés sur la figure du Aufhocker ont souvent tissé le lien entre cet être fantastique et l'Alp (F471.1. Nightmare (Alp). Presses person in dream) dans une tentative de comprendre l'origine de ce phénomène. Bien que dépassé dans son approche phénoménologique, ne tenant pas compte des contextes socio-historiques des narrations, le Handwörterbuch des deutschen Aberglaubens (HDA), publié entre 1927 et 1942, représente encore une source intéressante qui nous permet d'observer la tendance à tout classer qui se développe dans l'Allemagne des années vingt, quand le folklore commence à s'institutionnaliser en devenant une véritable discipline scientifique. Ainsi, l'imaginaire du Aufhocker est apparenté à l'imaginaire du cauchemar (Alpvorstellung) : cette expérience insolite de peur (Erlebnis der Angst) aurait donc une base psychophysique importante qui se manifeste dans le cadre d'une expérience numineuse (Numinose) sous la forme d'une angine de poitrine (Brustangst) (HDA : 675 ; cf. Ranke, 1922).

Nous constatons aussi que, dans les rapports d'expérience vécue par les individus ayant rencontré un être de type Aufhocker, cette entité numineuse a, dans 
un premier moment, des dimensions prodigieuses pour leur sauter, enfin, sur les épaules. Le sujet se sent comme paralysé, ne pouvant faire aucun mouvement volontaire. En outre, il ressent une lourde charge allongée sur son dos : cette " choses » sur les épaules ("Ding » auf den Schultern, DHA : 675) est interprétée comme un esprit (Spukgeist) qui lui serre la gorge. Leander Petzoldt (2003 : 7 ; 27-29) met en évidence la grande diversité des représentations phénotypiques de ces êtres surnaturels d'un être spirituel thériomorphe aux feux follets, en passant par des loups-garous, la chasse sauvage (cf. E501.15.3. Wild huntsman makes people carry bim on their backs) ou des génies des eaux (Hehmann, comme nos loups-garous aquatiques) - qui, dans leurs variations polymorphes, semblent répondre à une fonction analogue.

\section{Mirabilia orientales du monde arabo-persan}

Cans penser aux qarins de la tradition islamique des hadits, djinns qui nous rappellent le rôle des daimones grecs reposant sur les épaules de chaque humain afin d'influencer sa vie (Nasser, 2009 : 144), d'autres récits concernant des Aufhockers sont connus dans le domaine de la culture islamique arabo-persane. Pour identifier un être homologue à ceux que nous sommes en train d'analyser, nous devons suivre la migration d'une croyance à travers l'univers gréco-romain classique vers la Perse, sur la voie de la traduction pahlavi de la légende d'Alexandre le Grand (Meier, 1967), afin de rencontrer la figure persane du duwālpāy (forme arabe, duwāl-bāy), étymologiquement « être aux pieds à lanière en cuir ». Selon Nünlist (2015 : 265), les premières dénominations arabes de ces Aufhockers ${ }^{7}$ dateraient du $\mathrm{VIII}^{\mathrm{e}}$ siècle sous la forme de « al-qaffāzūn wa-ăl-hammāzūn » (ar. « sauteur sur le dos et éperonneur»), tandis qu'en persan la forme duwāl-pāy aurait déjà été attestée. Ces créatures, moitié humain et moitié non-humain (animal ou plante), habitent les arbres et, ayant des jambes très faibles, sautent sur les épaules d'une victime humaine et l'emprisonnent pour l'étrangler ou l'obliger à les emporter vers d'autres destinations (Nünlist, 2011 : 151-153).

Le folklore arabe nous permet aussi de mettre en évidence la figure de la g̀ul comme homologue du duwāl-pāy : dans son apparence de jolie femme, elle peut transformer ses victimes en ânes et les chevaucher. Ce comportement est similaire à celui des Alpfrau, ou Alpgeister, des gens turcs qui transforment ses victimes humaines en chevaux pour monter sur leurs dos lors de leurs pérégrinations nocturnes (ibid. : 153). Nous pouvons ainsi citer le motif narratif $\mathrm{G} 241.2$. Witch rides on person qui contribuera à intégrer nos réflexions concernant la nature composite de ces ontologies de type Aufhocker.

\section{La Kichkannī comme agent « aufhockeriseur » dans l'Himalaya népalais}

Tous terminons notre analyse en nous 1 penchant sur une figure du folklore des hautes castes hindouistes Bahun-Chhetri du Népal. La Kichkannī représente un $b \bar{a} y u$, manifestation dans le corps subtil de l'« âme " (ātman) d'une jeune fille vierge qui se serait suicidée. Esprit féminin d'une beauté élégante, elle peut acquérir la forme d'une fille séduisante, aux longs cheveux noirs qui retombent sur son sāri blanc, pour 
approcher des jeunes hommes et leur sucer le sang et les forces vitales. Elle développe ainsi des fonctions typiquement cauchemardesques (F471.2.1. Succubus : female incubus), se présentant, au cours de la nuit, auprès de sa victime pour venir peser sur la poitrine de celle-ci pendant son sommeil (Armand \& Cathiard, 2016). Si les narrations traditionnelles himalayennes concernant cet être surnaturel le représentent souvent dans sa nature d'autostoppeuse fantôme (E332.3.3.1. The Vanishing Hitchbiker), la Kichkanni peut aussi apparaitre comme un agent "aufhockeriseur " dans un document narratif que nous avons collecté à Thimi, au Népal :

Notre informateur nous raconte l'expérience qu'un de ses amis lui a rapportée. Une nuit, pendant qu'il était en train de rentrer chez lui, il a porté sur ses épaules une Kichkandì. Pendant la route, il avait perçu une présence blanche sur ses épaules mais, quand il s'est tourné pour mieux observer de quoi il s'agissait, la figure avait disparu. La Kichkandī est une figure habillée en blanc qui se fait transporter sur les épaules des personnes qui voyagent seules dans la nuit. (Document collecté auprès d'un informateur anonyme, Thimi - Bhaktapur, le 14 mars 2013) (E262. Ghost rides on man's back; E422.4.4 (a). Female revenant in white clothing. "The white lady")

\section{Fondements neuraux pour déceler l'identité de l'Autre numineux se portant sur le dos}

$\mathrm{L}$ es sections précédentes nous ont permis d'explorer transculturellement la variété d'ontologies surnaturelles pouvant revêtir la fonction d'Aufhocker. Nonobstant la richesse de ces narrations, nous pouvons individualiser une série d'éléments de base partagés par les sujets qui ont vécu une expérience de rencontre avec un agent " aufhockeriseur » : a) une présence indéniable se portant sur les épaules ou le dos ; b) une sensation de lourdeur écrasante qui peut parfois générer la c) paralysie du corps ; d) l'incapacité d'accomplir des mouvements volontaires. Nous proposerons par la suite une analyse de ces traits afin de pouvoir refonder neuralement l'identité des Aufhockers transculturels : ceux-ci deviennent des corps-fantômes bien réels. Nous viserons ainsi à démontrer comment l'agentivité et l'intentionnalité attribuées à ces êtres surnaturels peuvent être rattachées au rôle fondamental joué par la jonction temporo-pariétale (TPJ) de notre cerveau dans la génération neurale des ontologies qui hantent les imaginaires humains.

\subsection{Les apports de la paralysie du sommeil et de ses composantes, OBE (Out-of-Body Experience) et AP3S (Alien Presence Sensed from Self Shadowing)}

[...] je dormais sur le côté gauche, un peu en chien de fusil. Et... là ce qui s'est passé, c'est que à un moment y'a une... une forme noire, je me suis retrouvée avec une forme noire collée derrière moi dans le dos - je sais pas dire comment elle est arrivée - et avec des membres très souples, des bras et des jambes qui me... qui m'entouraient. Et... à un moment ça m'a serré très fortement, ça donnait vraiment l'impression d'un étouffement, 
d'un écrasement, ... au niveau ben des... du tronc, surtout des bras, du tronc et aussi au niveau des jambes. Et c'était, pendant quelques instants assez courts, ... comme une grande force qui mécrase. Et je pouvais pas du tout bouger, pas bouger d'un millimètre, j'avais l'impression d'être en plomb, quoi ! de... de peser très lourd, de rien pouvoir faire. [...] Et... ce qui était bizarre, c'est que... ben j'étais moi dans mon corps... avec... - je sentais ce truc, cette chose qui me serrait et en même temps, j'avais une vue du dessus, je voyais du dessus, ... je voyais la forme derrière moi. (Cathiard et al., 2012 : 48-49)

Le riche témoignage d'Eloïse Antzamidakis, informatrice privilégiée de Nicolas Abry, nous permet d'accéder à la narrativisation d'une expérience de paralysie du sommeil et de ses composantes fondamentales. Eloïse nous rapporte qu'elle perçoit une ombre noire, " collée derrière dans le dos », qui la serre désagréablement de ses " membres très souples »; elle peut aussi observer « ce truc, cette chose qui [la] serrait » d'une perspective extra-personnelle, en percevant avec une "vue de dessus " cette " forme derrière [elle] ». Nous retrouvons ici les deux composantes fondamentales de la paralysie du sommeil, ainsi que nous les avons présentées dans le modèle d'anthropologie neurocognitive BRAINCUBUS (Cathiard \& Armand, 2014) : une expérience hors-du-corps (OBE) et, ce qui nous intéresse tout particulièrement ici, une présence ressentie, décrite par l'acronyme AP3S (Alien Presence Sensed from Self Shadowing).

Nous reconnaissons ainsi une stricte parenté neurophysiologique entre les expériences de cauchemar, dans le sens étymologique d'esprit (germ. MAHR) qui piétine et opprime (lat. CALCARE) un dormeur, et d'êtres de type Aufhocker. Ces Autres Aliens deviennent des véritables corps "fantômes ", générés neuralement par l'activation de la jonction temporo-pariétale de l'hémisphère gauche de notre cerveau. La distinction " naïve » faite par de Blécourt entre les êtres qui étouffent pendant le sommeil (cauchemars) et les agents surnaturels qui attaquent les marcheurs nocturnes se posant de tout leurs poids sur le dos des passants n'est plus pertinente. Ignorant les bases neurophysiologiques qui ont généré ces deux expériences, de Blécourt ne reconnait pas qu'il s'agit de phénomènes homologues, au sens développemental, qui ont la même origine neurale. Il s'agit, dans les deux cas, de corps " fantôme ", de présences Aliens issues de l'ombre de notre Self.

\subsection{Des Autres Aliens dans la Jonction Temporo-Pariétale gauche}

$\Lambda$ fin de mettre en évidence les fondeexpériences de rencontre avec des ontologies surnaturelles, cauchemardesques ou " aufhockeriseuses ", nous devons avant tout préciser l'état cérébral d'AP3S. En 2006, lors d'un examen préopératoire en épileptologie, l'équipe suisse dirigée par le neurologue Olaf Blanke (Arzy et al., 2006) a découvert la sensation de présence d'un Alien, suite à la stimulation corticale d'une patiente épileptique de vingtdeux ans (sans problèmes psychiatriques, n'ayant jamais éprouvé cette expérience), dans la jonction temporo-pariétale gauche $e^{8}$, l'homologue du site des stimulations de 
l'expérience hors-du-corps (OBE) obtenue de manière répétée dans l'hémisphère droit (Blanke et al., 2002).

Suite à une première stimulation $(10.0 \mathrm{~mA})$, la patiente, allongée en position supine, rapportait l'impression qu'il y avait quelqu'un derrière elle. D'autres stimulations (10.0-11.0 mA) ont induit l'expérience décrite par la patiente comme la sensation qu'une « ombre ", qui ne parlait et ne bougeait pas, se trouvait derrière elle, sur son dos, dans la même position'. Une autre stimulation $(11.0 \mathrm{~mA})$ est induite avec la patiente assise entourant ses jambes de ses bras : la figure est elle aussi assise dans la même position et serre désagréablement la femme de ses bras ("the "man" was now also sitting and he was clasping her in his arms, which she described as an unpleasant feeling »). Enfin, des stimulations $(11.0 \mathrm{~mA})$ ont été appliquées alors que la patiente était en train d'accomplir une tâche de dénomination en tenant une carte dans la main gauche : cette fois-ci, la figure se trouvait assise derrière elle, un peu déplacée sur sa droite, et cherchait à interférer dans l'exécution de la tâche ("she again reported the presence of the sitting "person", this time displaced behind her to her right and attempting to interfere with the execution of her task ("He wants to take the card"; "He doesn't want me to read")»).

En raison de la contre-agentivité propre à cette présence, la patiente ne reconnaît pas que l'ombre, qui copie ses mouvements et les contrarie, n'est qu'une illusion de son propre corps. Cette attitude, étudiée chez une patiente non-délusionnelle, est pourtant emblématique des patients schizophréniques délusionnels qui, à cause d'une hyperactivité dans le cortex temporo-pariétal, attribuent leurs propres actions à quelqu'un d'autre (Farrer et al., 2004). Cette expérience de "présence ressentie " induite dans le TPJ gauche est issue d'une dissociation agentive qui génère clairement un corps "fantôme " contre-agentif, un corps "fantôme " Alien qui s'oppose au corps-physique du sujet encore habité par 1e Self. À cause du caractère manifestement alien, étranger et hostile de ce corps " fantôme ", pourtant venu du Self, il n'est pas reconnu comme lui appartenant.

À partir des études novatrices de topographique corticale conduites par Wilder Penfield, à partir des années trente, il a été possible de décrire un bomonculus sensori-moteur, une figure localisée dans la zone corticale au niveau du sillon central, séparant le lobe frontal du pariétal, représentant les différentes parties du corps humain. Les avancées des connaissances sur le cortical mapping ont enfin permis d'établir qu'une carte complète de notre corps, enregistrée dans ce sillon interémisphérique, serait normalement " unie » (matched) aux limites périphériques de notre corps physique. Avec l'activation de la jonction temporo-pariétale gauche, nous assistons à une dissociation entre le corps physique et celui parasomatique, les deux appartenant au Self du sujet. Ainsi, lors de cette expérience, le Self du sujet reste, pour ainsi dire, enveloppé dans le corps physique sans reconnaitre que le corps parasomatique, ce corps "fantôme » dissocié et projeté à l'extérieur des limites de son corps physique, lui appartient. Comme nous l'avons déjà indiqué, le sujet l'interprète donc comme étant un être Alien bien réel. Nous pouvons ainsi interpréter ces expériences dans le cadre de connectomes, de cartes de réseaux neuraux, de corps-fantômes neuralement bien réels. 


\subsubsection{Des connectomes de corps dissociés vers les expériences autoscopiques}

$\mathrm{C}$ onscient que la paralysie du sommeil représente l'état le mieux partagé par des homéothermes, parmi lesquels nous trouvons les humains où nous avons le plus de chance (en prévalence) d'éprouver l'expérience de connectomes de corps dissociés, ces êtres Aliens ne se manifestent pas uniquement au cours d'expériences de paralysie du sommeil, quand le sujet est allongé dans son lit, ou suite à des stimulations corticales préopératoires en laboratoire. Comme nous l'avons déjà montré dans Armand \& Cathiard (2019 : 242-243), ils peuvent aussi bien accompagner Reynold Messner dans la descente par le versant Diamir du Nanga Parbat ou, plus récemment, venir offrir une coupe de thé chaud à une autre alpiniste, Elisabeth Revol, qui, après avoir dû abandonner son compagnon, Tomasz Mackiewicz, sur cette même " montagne nue » de l'Himalaya pakistanais, cherche à redescendre vers la vallée dans des conditions extrêmes. Ces sujets, pourtant sains et en pleine activité physique, rapportent bien des présences indéniables qui interagissent avec eux, en se limitant à les suivre - « elle est simplement là ", nous dit Messner, « elle s'arrête quand je m'arrête, se remet en mouvement quand je bouge »ou développant des véritables interactions complexes où il est possible de reconnaître une intention, positive ou malfaisante, dans leurs comportements.

Nous avons ainsi mis en évidence que ces expériences peuvent être situées du point de vue neuronal dans le cadre des phénomènes autoscopiques, des désordres dans la perception et/ou dans la conscience du corps propre liés à des processus de désintégration multisensorielle ou sensorimotrice ou à des variations systématiques des fonctions vestibulaires. Le rôle de la jonction temporo-pariétale serait encore une fois fondamental pour le déclanchement de ces expériences autoscopiques (Sforza \& Blanke, 2012).

\subsection{Agentivité et intentionnalité : quelle identité pour nos agents " aufhockeriseurs"?}

Tous avons constaté que les ontologies 1 surnaturelles que nous avons présentées dans cette étude sont décrites comme des agents intentionnels. L'attribution d'une agentivité suppose que l'être agent décide intentionnellement de commencer une action sur la base de ses propres impulsions internes et non pas seulement d'une façon mécanique, en répondant à des événements de l'environnement où il se trouve. Nous rappelons à titre d'exemple que la dame rencontrée par Elisabeth Revol sur le Nanga Parbat lui propose un échange ayant un but précis : du thé chaud contre une chaussure ${ }^{10}$. Afin de pouvoir être reconnu comme agent réel, il est donc nécessaire de lui attribuer une intentionnalité, la capacité d'établir une relation active avec un objet ou une autre entité présents dans la réalité. Pour interagir convenablement, les humains ont développé la capacité de comprendre consciemment les intentions des autres agents avec lesquels ils entrent en contact. Pour cela, ils possèdent un ensemble de dispositifs cognitifs, appelé théorie de l'esprit (Theory of Mind), qui permettent la construction de la représentation des autres et la compréhension de leurs comportements. 
Nous avons vu que la " chose » qui tombe sur les épaules du marcheur nocturne rentrant de Brantôme a été décrite comme étant quelque chose de « mou, lourd et [qui] respirait comme un être vivant». Cette entité, un lèbérou, est donc présentée comme ayant une existence propre et une corporéité matérielle, décrite par le sujet en termes de pesanteur écrasante et mollesse tactile et enveloppante.

Une autre caractéristique de l'expérience d'Aufhocker est, comme nous l'avons montré, l'incapacité d'accomplir des mouvements volontaires, voire la sensation que cet agent corporel puisse prendre le contrôle volontaire du corps de sa victime, en forçant, par exemple, un des protagonistes de nos récits «à se diriger vers la rivière toute proche ", emportant "par une force irrésistible " l'homicide qui avait refusé de répondre au monitoire ou encore se laissant simplement transporter sur les épaules dans les ruelles de Thimi, au Népal. Nous reconnaissons donc que ces agents « aufhockeriseurs » développent une intentionnalité propre qui s'oppose à la volonté des victimes impuissantes sur lesquelles ils s'installent.

Le modèle pariéto-frontal sur la cognition motrice de Desmurget et Sirigu (2009; Desmurget et al., 2009 ; 2018) nous permet d'opérer une distinction importante entre l'intention motrice (conscious motor intention) et le sentiment moteur (motor awareness). Lors des expériences de stimulation corticale, les sujets stimulés dans le cortex pariétal postérieur (PPC) rapportent avoir ressenti l'envie, l'intention motrice consciente, de vouloir bouger certains membres de leur corps - et cela selon le locus de la carte somatotopique dont nous avons déjà parlé. En augmentant légèrement le voltage de la stimulation, ils ont l'impression d'avoir réellement bougé leur membre (n'ayant pas accès visuel ou tactile à leur membre), en pure motor awareness, alors qu'aucun mouvement n'avait été enregistré, même pas la contraction des muscles. Une des réactions d'un sujet suite à une stimulation dans l'aire de la face est particulièrement intéressante : I I moved my mouth, I talked, what did I say? " (Desmurget et al., 2009 : 812). Cependant, lorsqu'on leur montre cette absence de motricité, ils sont plutôt surpris, mais, nétant pas des sujet délusionnels, ils acceptent cette réalité.

Ces réflexions peuvent alors nous aider à comprendre le dernier des traits fondamentaux de l'expérience d'Aufhocker : l'incapacité du sujet d'accomplir des mouvements volontaires et la sensation conséquente que lêtre "aufhockeriseur » prend le contrôle de son corps pour le diriger, selon son gré, où il souhaite. Comme nous l'avons montré pour les autostoppeuses fantômes des Alpes et de l'Himalaya (Armand \& Cathiard, 2019 : 244-245), les sujets « aufhockerisés » n’ont pas conscience de faire un mouvement volontaire car ce dernier a été généré dans le seul lobe frontal de la motricité sans la coordination avec le lobe pariétal de l'intention motrice : ils l'attribuent donc à un être agent, une ontologie " aufhockeriseuse ", dont ils ressentent la présence indéniable sur leur dos.

\section{dים}

Afin de définir l'identité de ces ontologies de type Aufhocker en tant qu'entités perçues comme existant dans la réalité par les sujets qui en ont fait l'expérience, nous 
avons mis en évidence différents aspects de leur nature. Premièrement, nous avons constaté que ces êtres présentent une corporéité matérielle - un corps qui écrase - et une extension spatiale ${ }^{11}$ : il s'agit en effet de véritable corps "fantômes " venant peser sur la poitrine des dormeurs ou montant sur le dos des marcheurs nocturnes. En tout cas, ces ontologies semblent bien occuper une portion de l'espace extrapersonnel et interagissent tactilement (sensation de pesanteur, de mollesse, d'être enveloppé par leurs membres, etc.) avec les sujets rapportant ce type d'expériences.

En outre, en reprenant l'expression de Cheyne et Girard (2007 : 985, nos mises en évidence), l'expérience vécue d'une rencontre avec un Aufhocker peut être décrite comme " $a$ feeling of the presence of a Being, and not as a mere existent thing, but an intentional Being with a mind and a soul $\aleph^{12}$. Nous reconnaissons ainsi les domaines de l'agentivité et de l'intentionnalité comme étant propres à la nature de ces êtres de l'imaginaire humain. Ces corps «fantômes » expérientiels, qui ne sont pas reconnus comme appartenant à notre Self, deviennent ainsi de véritables systèmes d'action et d'intention. Le modèle pariéto-frontal de Desmurget et Sirigu nous est venu en aide pour encadrer du point de vue neural la perte du contrôle moteur rapportée par les sujets « aufhockerisés ».

Enfin, nous devons reconnaître le rôle fondamental de la jonction temporo-pariétale du cerveau humain dans la génération de tous ces Autres Aliens (contre-)agentifs. Le TPJ devient notre pivot neuronal des processus de traitement des informations concernant le Self en relation avec les informations multisensorielles concernant le corps. Ces processus d'intégration multisensorielle au niveau de TPJ permettent ainsi d'étudier la localisation d'ontologies dans l'espace environnant, en leur attribuant la capacité de percevoir et d'agir la réalité. Ainsi, l'excellente revue de Wible (2012) nous montre l'important rôle joué par TPJ dans les différents aspects de la cognition sociale, comme l'empathie et la théorie de l'esprit, mais aussi dans la perception de l'agentivité et l'intentionnalité.

\section{BibliograPHIE}

Fabio Armand, "Entre-corps chamaniques tremblants : Self et corps-fantômes Aliens dans quelques rituels d'incorporation d'ontologies numineuses dans les chamanismes de l'Himalaya népalais ", in Fintz C. (dir.), L'observation de l'entre-corps : imaginaire et émotions, Valence, Presses Universitaires de Valenciennes, 2020, p. 153-170.

Fabio Armand, «Le loup-garou : de sa liminalité dans le patrimoine narratif gallo-roman à sa parenté neuro-anthropologique ", in Reusser-Elzingre A. \& Diémoz F. (dir.), Le patrimoine oral : ancrage, transmission et édition dans l'espace galloroman, Berne, Peter Lang, 2016, p. 175-192.

Fabio Armand, « Les loups-garous et les eaux », in IRIS, « Hommage à Gilbert Durand », n. 34, 2013, p. 133-145.

Fabio Armand \& Marie-Agnès Cathiard, « Quand notre moi se fait passer incognito pour un Autre », in Caietele Equinox, «Imaginaire de l'altérité. I. Pour une approche anthropologique », vol. 36, 2019, p. 235-247.

Fabio Armand, Marie-Agnès Cathiard \& Christian Abry, « Neuronuminous-like Experiences within the BRAINCUBUS Framework for Cognitive Folkloristics: Present Promise and Limits ", in Numinoses Erzäblen: Das Andere - Jenseitige - Zauberische, Deutsche Gesellschaft für Volkskunde, 2019, p. 57-65. 
Fabio Armand, Marie-Agnès Cathiard \& Christian Abry, « Beyond nationalistic isolationisms: our species' brain dreams and nightmares. Re-embodying daemonic ontologies in the Mediterranean South of the Alps for this XXIth century », in K. Jurčević Katica, L. Kaliterna Lipovčan, \& O. Ramljak (éds), Imaging the Mediterranean: Challenges and Perspectives, Institute of Social Sciences Ivo Pilar et VERN Group, , 2017, p. 113-125.

Shahar Arzy, Margitta Seeck, Stephanie Ortigue, Laurent Spinelli, \& Olaf Blanke, "Induction of an illusory shadow person", in Nature, vol. 443, 2006, p. 287-288.

Jörg Bartscher-Kleudgen, Die Schwarzen Führer - Eifel, Eulen Verlag, 2002.

Richard Beitl, Im Sagenwald. Neue Sagen aus Vorarlberg, Feldkirch, 1953.

Willem de Blécourt, "The Differentiated Werewolf: An Introduction to Cluster Methodology", in Willem de Blécourt (éd.), Werewolf histories, Palgrave MacMillan, 2015: 23-43.

Willem de Blécourt, "I Would Have Eaten You Too": Werewolf Legends in the Flemish, Dutch and German Area", in Folklore, vol.118, 2007, p. 23-43.

Marie-Agnès Cathiard, Nicolas Abry \& Christian Abry, «A propos des récits de corps fantômes dans la paralysie du sommeil. Existe-t-il un pont psychologique neurocompatible entre l'expérience d'une illusion et l'adhésion à une délusion ? ", in Serclus, vol 2, 2012, p. 47-72.

Marie-Agnès Cathiard \& Fabio Armand, «Braincubus : vers un modèle anthropologique neurocognitif transculturel pour les «fantômes » de l'imaginaire », in P. Pajon \& M.-A. Cathiard (éds), Les imaginaires du cerveau, Fernelmont, IEME, 2013, p. 53-87.

Allan J. Cheyne \& Todd A. Girard, "The nature and varieties of felt presence experiences: A reply to Nielsen”, in Consciousness and Cognition, vol. 16, 2007, p. 984-991.

Maurice Crampon, Le culte de l'arbre et de la forêt en Picardie. Essai sur le folklore picard, Paris, A. Picard, 1936.

Michel Desmurget \& Angela Sirigu, «A parietal-premotor network for movement intention and motor awareness ", in Trends in Cognitive Science, vol. 13, n. 10, 2009, p. 411-419.

Michel Desmurget, Nathalie Richard, Pierre-Aurelien Beuriat, Alexandru Szathmari, Carmine Mottolese, Jean-René Duhamel \& Angela Sirigu, "Selective inhibition of volitional hand movements after stimulation of the dorsoposterior parietal cortex in Humans", in Current Biology, vol. 28, 2018, p. 1-7.

Michel Desmurget, Karen T. Reilly, Nathalie Richard, Alexandru Szathmari, Carmine Mottolese \& Angela Sirigu, "Movement Intention After Parietal Cortex Stimulation in Humans ", in Science, vol. 324, n. 5928, 2009, p. 811-813.

Chloe Farrer, Nicolas Franck, Chris D. Frith, Jean Decety, Nicolas Georgieff, Thierry d'Amato \& Marc Jeannerod, "Neural correlates of action attribution in schizophrenia ", in Psychiatric Research: Neuroimaging, vol. 131, 2004, p. 31-44.

Jean Fleury, Littérature orale de la Basse-Normadie (Hague et Val-de-Saire), Paris, Maisonneuve et C. éditeurs, 1883.

Gerda Grober-Glück, « Aufhocker und Aufhocken nach den Sammlungen des Atlas der deutschen Volskunde », in Rheinisches Jarbuch für Volkskunde, n. 15/16, 1965, p. 117-143.

HDA: Hanns Bächtold-Stäubli, Handwörterbuch des deutschen Aberglaubens, 10 vols, Berlin, Walter de Gruyter, 1927-1942.

András Höfer, Tamang Ritual Texts I - Preliminary studies in the folk-religion of an ethnic minority in Nepal, Wiesbaden, Franz Steiner Verlag, 1981.

Claude Lecouteux, Au-delà du merveilleux. Essai sur les mentalités du Moyen Âge, Presses de l'Université Paris-Sorbonne, 1998.

Ioan M. Lewis, Ecstatic religion. A study of shamanism and spirit possession, Penguin books, 1971.

Fritz Meier, « Das Volk der Riemenbeinler », in Festschrift Wilhelm Eilers, Wiesbaden, Harrasowitz, p. 341-367.

Thomas Metzinger, Being No One, Cambridge, MIT Press, 2003.

Konrad Müller, Die Werwolfsage: Studien zum Begriff der Volkssage, Karlsruehe, Macklotsche Verlag, 1937. 
Lara Nasser, "The Jinn. Companion in the Realm of Dreams and Imagination » in K. Bulkeley, K. Adams \& P. M. Davis, Dreaming in Christianity and Islam. Culture, Conflict and Creativity, Rutgen University Press, 2009, p. 143-154.

Tobias Nünlist, Dämonenglaube im Islam, Berlin, de Gruyter, 2015.

Tobias Nünlist, «Von Berittenen und Gerittenen: Aspekte des Dämonenglaubens im Bereich des Islams », in Asiatische Studien, vol. LXV, p. 145-172.

Leander Petzold, Kleines Lexikon der Dämonen und Elementargeister, München, Verlag C. H. Beck, 2003.

Friedrich Ranke, «Der Huckup », in Bayerische Hefte für Volkskunde, n. 9, 1922, p. 1-33.

Fons Roeck, « Nederlandse weerwolfsage in de negentiende en twintigste eeuw. » Unpublished Ph.D. diss., Catholic University of Leuven 1967.

Paul Sébillot, Le folklore de France-deuxième tome : La mer et les eaux douces, Paris, E. Guilmoto éditeur, 1904.

Paul Sébillot, Traditions et superstitions de la Haute-Bretagne, vol. 1, Paris, Maisonneuve et C. éditeurs, 1882.

Claude Seignolle, Les Evangiles du diable selon la croyance populaire - Le grand et le petit Albert, Paris, Robert Laffont, 1998.

Anna Sforza \& Olaf Blanke, « Autoscopic Phenomena: Clinical and Experimental Perspectives », in Jan Dirk Blom \& Iris E. C. Sommer (éds.), Hallucinations. Research and Practice, New York: Springer, 2012, p. 187-201.

Stith Thompson, Motif-Index of Folk-Literature, Bloomington, Indiana University Press, 1966.

Cynthia Gayle Wible, "Schizophrenia as a Disorder of Social Communication ", in Schizophrenia Research and Treatment, 2012, p. 1-12.

Maeike, Zijlmans, Pieter van Eijsden, Cyrille H. Ferrier, Kuan H. Kho, Peter C. van Rijen, \& Frans S. S. Leijten, «Illusory shadow person causing paradoxical gaze deviations during temporal lobe seizures ", in Journal of Neurology, Neurosurgery E' Psychiatry, vol. 80, 2009, p. 686-688.

\section{Notes}

1. Les narrations que nous présentons dans cette étude seront classées en utilisant le Motif-Index of Folk-Literature de Stith Thompson (1966), œuvre monumentale pour toute enquête comparative dans le domaine de la folkloristique narrative.

2. Nous n'analyserons pas ici les narrations où sont présentées des expériences de possession (ex. "the spirit when incarnated in its heartly host is said to ride its host (...) the spirits "mount" them and they also "mount" the spirit", chez les Haussa de l'Afrique occidentale et les Sidamo de l'Afrique méridionale : cf. Lewis, 1971 : 51) et d'incorporation d'entités numineuses dans les rituels chamaniques (Armand, 2020). Pour n'en donner qu'un exemple, nous rappelons que les bompos-chamans du groupe ethnique des Tamang (Himalaya népalais) utilisent la construction « gori yuba " (tamang: go < tibétain : $k h o g$, « le corps et la partie postérieure du corps, le dos ») pour décrire l'incorporation d'un esprit à l'intérieur du corps physique : l'entité numineuse descend sur le dos du chaman (Höfer 191 :31), se laissant " porter sur [s] on dos par derrière " (gyabna li:jye khurba).

3. «Un Etre (...) qui n'est pas une simple chose existante, mais un Être intentionnel».

4. Si ces récits présentent des Aufhockers qui jouent un rôle malfaisant, les ménétriers qui ont la malchance de rencontrer un loup sur leur chemin peuvent jouer du violon afin de sauver leur vie en faisant danser, avec leur musique, des lutins sur le dos du loup menaçant : « (...) les lutins, charmés par le son de violon, accoururent pour se livrer à leur occupation favorite, la danse. L'un d'eux enfourcha le loup et le fit danser pendant une heure, jusqu'à ce qu'il meure. Son cadavre fut jeté dans un taillis. Les génies avaient aidé l'homme à se débarrasser du démon. Le ménétrier joua encore une ronde et les lutins dansèrent sur les branches, les feuilles et les fleurs sans faire ployer les végétaux. » (Crampon, $1936: 162$ ) 
5. Nous trouvons aussi des formes non lupines d'Aufhocker dans la Volkskunde du Vorarlberg autrichien : dans le domaine de la sorcellerie, une femme fait apparaitre un poids épouvantable sur le dos d'un certain Josef Bilgeri qui rentrait chez lui, à Lochau (Beitl, 1953 : 45, n. 33), tandis qu'un autre homme accepta de porter sur son dos un fantôme errant (Wanderer geist) jusqu'à l'église de Rankweil pour lui permettre de se racheter devant Dieu : il en mourra quelques semaines plus tard, après avoir porté ce fardeau surnaturel (unheimlichen Bürde) (Ibid: 91, n. 131). Nous mentionnons également l'histoire de Babkauv aquatique d'Aachen, de la Rhénanie septentrionale : cet être à la fourrure hirsute, aux dents acérées et aux yeux brillants, serait sorti de l'ancien canal de drainage des sources Kaiser et Büchel pour se faire transporter sur les épaules par les voyageurs nocturnes (Bartscher-Kleudgen, $2002: 23$ ).

6. «Roeck considère le loup-garou « qui saute sur le dos » comme un exemple de la désintégration de la légende du loup-garou, et de l'effacement des frontières entre le loup-garou et les autres figures. Sa conclusion «qu'à l'origine, la chevauchée sur le dos des victimes nétait pas une partie de la légende du loup-garou» fait écho à la thèse de Muller selon laquelle il s'agissait d'une nouvelle composante, tirée du cauchemar. Cet argument est cependant incorrect, car le cauchemar est censé étouffer les gens lorsqu'ils sont couchés dans leur lit, et non lorsqu'ils se promènent dans la nuit ».

7. Nünlist (2011) regroupe ces ontologies fantastiques sous les termes allemands d'Aufhocker et de Riemenbeinler, « cravacheur », du verbe " cravacher », en équitation, cingler avec une cravache.

8. Cette même expérience a été répliquée plus récemment chez un autre sujet épileptique par Zijlmans et al. $(2009$, p. 686)

9. «He was bebind me, almost at my body, but I do not feel it »: il est intéressant de constater ici que la patiente utilise le pronom personnel masculin de la troisième personne, "he ", et non pas un pronom générique neutre, «it», pour décrire cette présence. Nous constaterons aussi que, dans les autres rapports de sensation qui font suite à des nouvelles stimulations corticales dans la région du TPJ, la patiente verbalise son expérience en utilisant les termes " man » (" homme ») et "person » ("personne») pour décrire cette présence ressentie.

10. Tiré de notre transcription d'une interview d'Elisabeth Revol pour France Télévision, le 31 janvier 2018 : «j'imaginais des personnes qui m'amenait du thé chaud, des bouteilles d'eau chaude et, à un moment donné, au fait, pour remercier ces personnes il y a une dame qui me dit "Mais est-ce que je peux prendre ta chaussure ? Parce que voilà je t'ai amené quelque chose de chaud et maintenant tu te sens mieux... et alors est-ce que je peux prendre ta chaussure ? ". Et, à ce moment, au fait, machinalement, je me lève, j'enlève ma chaussure et je lui donne ma chaussure. Et le matin, au fait, je me suis levée et j'avais simplement la chaussette et la chaussure était tombée dans la crevasse ».

11. À ce propos, nous avons déjà corrigé (Armand et al., $2017: 120-122$ ) une erreur issue du travail de Thomas Metzinger qui, contribuant au développement d'une neurophilosophie de l'âme, met en évidence, dans le cadre des phénoménologies des expériences hors-du-corps, un " second corps invisible et sans poids, mais étendu dans l'espace» (2003:55 notre traduction ; nous soulignons).

12. «La sensation de présence d'un $\hat{E} t r e$, et pas comme une simple chose existante, mais comme un Être intentionnel avec un esprit et une âme». 